SWGRI/SinoPec

jge-2d3c-tang-rev2.doc

Nov 2007

\title{
PP and PS Seismic Response from Fractured Tight Gas Reservoirs: A Case Study
}

Tang Jianming ${ }^{1,2}$, Zhang Shaonan ${ }^{1}$ and Xiang-Yang $\mathrm{Li}^{3}$

${ }^{1}$ College of Energy Resources, Chengdu University of Technology, Sichuan, China

${ }^{2}$ SWGRI,SINOPEC, No.398 South Taishan Road, Deyang, Sichuan, China, 618000

${ }^{3}$ British Geological Survey, West Mains Road, Edinburgh EH9 3LA, UK

Suggested short title: PP and PS Response from Fractured Reservoirs

Intended publication: Journal of Geophysics and Engineering

Submitted on 27 April 2007

$1^{\text {st }}$ Revised on $\quad 10$ September 2007

$2^{\text {nd }}$ Revised on $\quad 7$ November 2007

*Address for correspondence

Xiang-Yang Li

Britigh Geological Survey

Murchison House,

West Mains Road

Edinburgh EH9 3LA

Scotland,UK

xyl@bgs.ac.uk 


\begin{abstract}
In this paper, we present an example of using PP and PS converted-wave data recorded by digital MEMS (micro-eletro-mechanical-system) to evaluate a fractured tight gas reservoir from the Xinchang gas field in Sichuan China. For this, we analyze the variations in converted shear-wave splitting, Vp/Vs ratio and PP and PS impedance, as well as other attributes based on absorption and velocity dispersion. The reservoir formation is tight sandstone, buried at a depth of about $5000 \mathrm{~m}$, and the converted-wave data reveal significant shear-wave splitting over the reservoir formation. We utilize a rotation technique to extract the shear-wave polarization and time delay from the data, and a small-window correlation method to build time-delay spectra that allow the generation of a time-delay section. At the reservoir formation, the shear-wave time-delay is measured at $20 \mathrm{~ms}$, about $15 \%$ shear-wave anisotropy, correlating with the known gas reservoirs. Furthermore, the splitting anomalies are consistent with the characteristics of other attributes such as $\mathrm{Vp} / \mathrm{Vs}$ ratio and $\mathrm{P}$ - and S-wave acoustic and elastic impedance. The P-wave shows consistent low impedance over the reservoir formation, whilst the S-wave impedance shows relatively high impedance. The calculated gas indicator based on absorption and velocity dispersion yields a high correlation with the gas bearing formations. This confirms the benefit of multicomponent seismic data from digital MEMS sensors.
\end{abstract}

Keywords: multicomponent, converted-waves, fractured gas reservoir

\title{
Introduction
}

The use of converted-wave seismic data for reservoir characterization can be trace back to the 1970's, and Stewart et al. (2002) and Li et al. (2007) gave a good overview on the development of this technology. Despite an increased interest from the hydrocarbon industry, the anticipated breakthrough into mainstream technology is still to be realized. One of the problems is the lack of good quality data that demonstrate the full benefit of the technology. This is particularly true for land multicomponent seismic. Recently, the use of digital MEMS (micro-electro-mechanical system) sensors has substantially improved the quality of land converted-wave data (Calvert et al., 2005). As a result, here we present an example of using 
MEMS-based converted-wave technology for evaluating fractured tight-sandstone gas reservoirs from the Sichuan gasfield in Southwest China.

The Sichuan province is one of largest gas provinces in China, and gas production in Sichuan is mainly from deep buried tight sandstone and carbonates at depth from $4000 \mathrm{~m}$ to $6000 \mathrm{~m}$. Fracture characterization is critical for ensuring economic gas production from these tight formations of otherwise low permeability. The use of P-wave techniques for characterising these fractures has not been very successful in this area due to the depth limitations. Previous multicomponent seismic experiments have also had limited success due to data quality issues. The advent of MEMS sensors renewed the interest to use PP and PS multicomponent seismic data in this area. As a result, new multicomponent experiments have been carried out. Three 2D3C test lines were acquired during 3D3C multicomponent experiments conducted in 2005, and the analysis results of test lines are presented in this paper.

\section{Geological Background}

The study area is located in the Sichuan Province in Southwest China. The ancient inclines formed in the Indosinian and Yanshanian periods are good traps for gas movement and accumulation. Early reservoirs were enclosed in deep tight sandstones. Tectonic movement and fracturing caused by uplift and pressure deformation in the Himalayan Period make some early gas deposits migrate to the upper Jurassic formation and accumulate along faults and fractures in the formation, forming many new reservoirs. Meanwhile the early gas reservoirs in the deeper formations were activated and re-grouped into places where porosity and permeability were relatively higher and fracture growth was intensive, and finally new and rich reservoirs were formed such as the Xinchang field near Chengdu, the provincial capital of Sichuan.

Figure 1 shows the stratigraphy of the gas reservoirs in Xinchang field. The target formation is at around 5,000 meters depth from the surface. Petrophysical analysis shows that the average matrix porosity is less than $3 \%$, average matrix permeability is less than $0.1 \times 10^{-3}$ $\mu \mathrm{m}^{2}$ for more than $55 \%$ of the core samples taken from the field, as shown in Figure 2. Consequently, these reservoirs are classified as deep buried tight sandstone reservoirs with extremely low porosity and permeability. 


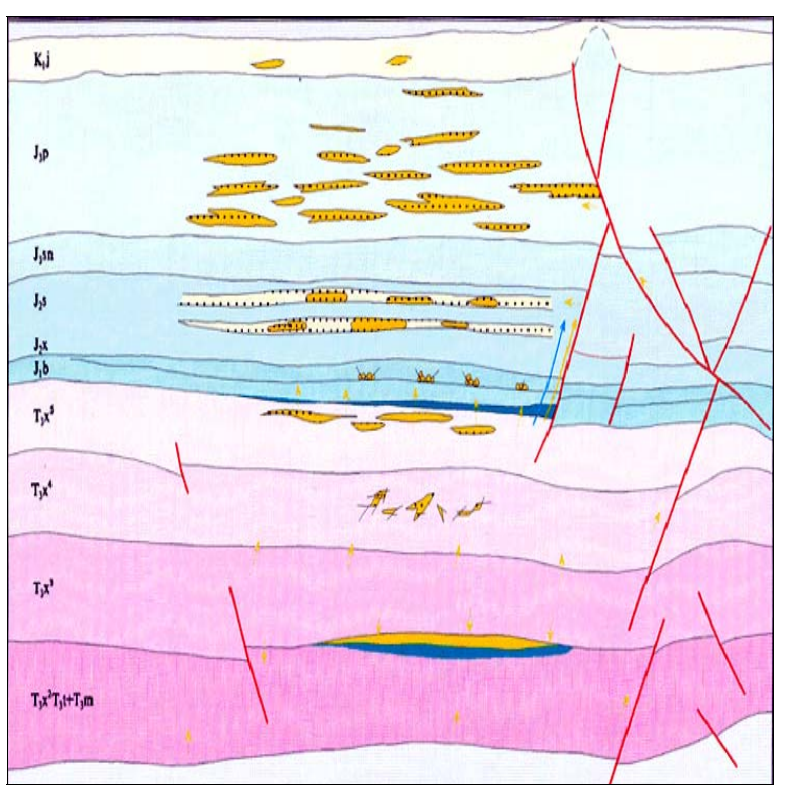

(a) Reservoir pattern

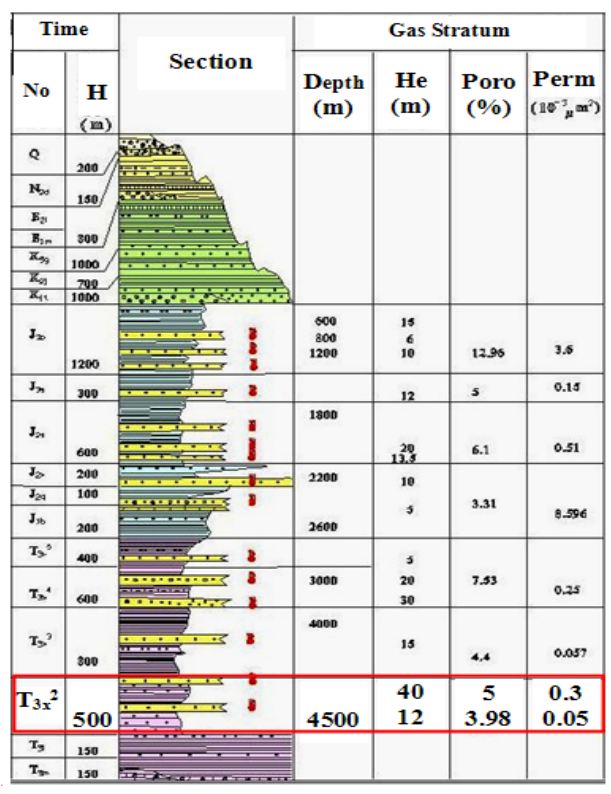

(b) stratigraphy

Figure 1: (a) A typical geological section showing the gas reservoir pattern and (b) the stratigraphic column, where $T_{3 x} 2$ marks the target formation.

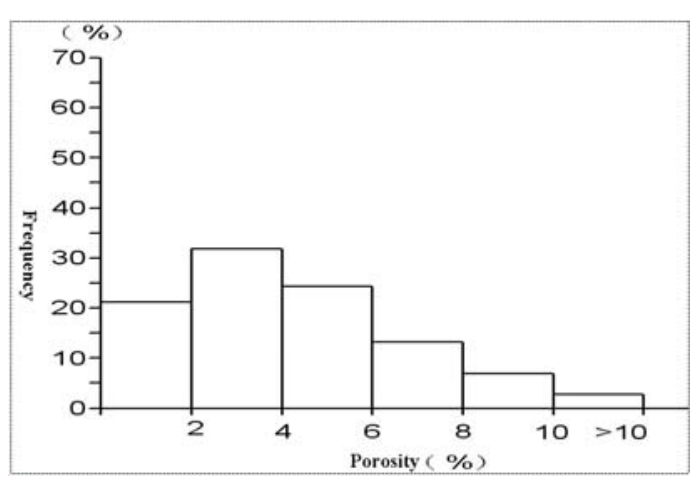

(a) Porosity

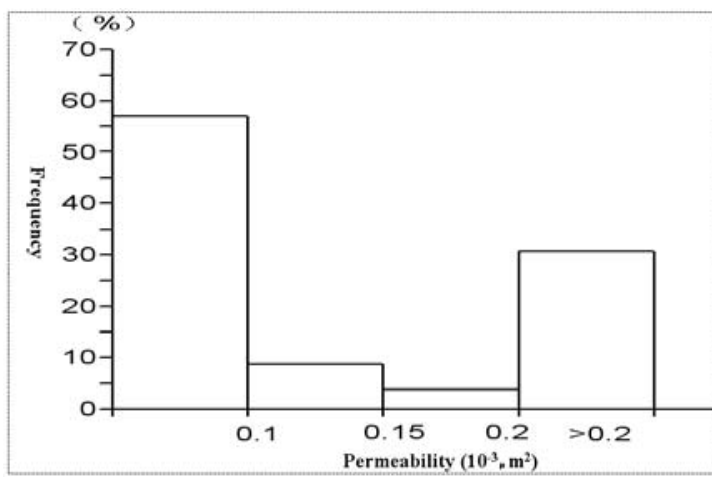

(b) Permeability

Figure 2: (a) Porosity and (b) permeability distribution in the core samples taken from the study areas.

The presence of near vertical fractures, giving rise to horizontal transverse isotropy (HTI), is critical to ensure economic production in the Xinchang Gas Field. As shown in Figure 3, core samples reveal intensive fracturing in the target formation. Table 1 compares the distribution of low and high angle fractures with productivities from four boreholes in the Xinchang field, confirming the importance of the presence of near vertical high-angle fractures. 


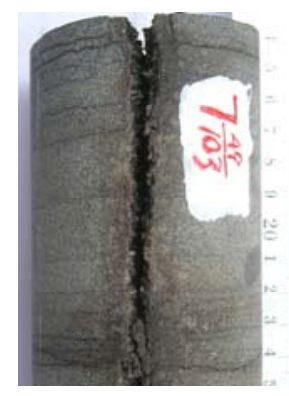

(a) Well X565

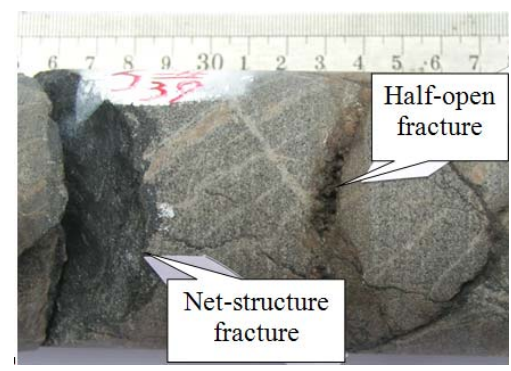

(b) Well X853

Figure 3: Core samples in wells (a) X565 and (b) X853 in the Xinchang Gas Field, revealing heavy fracturing.

Table 1: Correlation of gas productivities with fracture density of low and high angle fractures for four wells in the study area.

\begin{tabular}{|l|c|c|c|}
\hline Wells & $\begin{array}{c}\text { Density of low } \\
\text { angle fracture } \\
\text { (number/m) }\end{array}$ & $\begin{array}{c}\text { Density of high } \\
\text { angle fracture } \\
\text { (number/m) }\end{array}$ & $\begin{array}{c}\text { Productivity } \\
(\mathrm{AOF} \times 104 \mathrm{~m} 3 / \mathrm{d})\end{array}$ \\
\hline CH138 & 14.03 & 2.25 & Pygmaean \\
\hline CH140 & 10.06 & 0.43 & No gas \\
\hline CH127 & 8.51 & 15.1 & 19.245 \\
\hline CH137 & 2.84 & 7.74 & 33.8044 \\
\hline
\end{tabular}

\section{Data acquisition and characteristics}

The experiment consisted of three 2D lines with about $45 \mathrm{~km}$ of full-fold coverage, as shown in Figure $4 \mathrm{a}$, where the red box shows the 3D coverage. The three lines are located in the centre of the study area that is relatively flat (Figure 4b). Line 1(L1) and Line 2(L2) are orthogonal to each other, and Line 3(L3) is at approximately $45^{\circ}$ to L1 and L2. The three lines form an intersection point at well X851, which is the most productive well in the region. Multicomponent VSPs had also been acquired from the boreholes for helping in correlating the PP- and PS-sections, together with some P- and S-wave sonic logs. To aid statics correction for converted-waves, refraction surveys (blue points, Figure 4c) and multicomponent uphole (red points) were conducted during acquisition (Figure 4c). All the lines were acquired with a split spread with a maximum offset of $8640 \mathrm{~m}$, nominal receiver interval of $40 \mathrm{~m}$, and shot interval of $120 \mathrm{~m}$. Table 2 lists all the acquisition parameters. The main purpose of the test lines is to evaluate converted-wave shear-wave splitting and acquisition parameters such as source magnitude, geophone type, etc. 


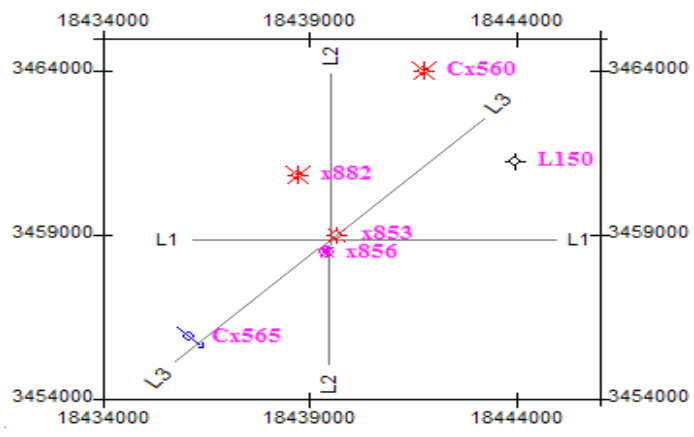

(a) Layout of the test lines

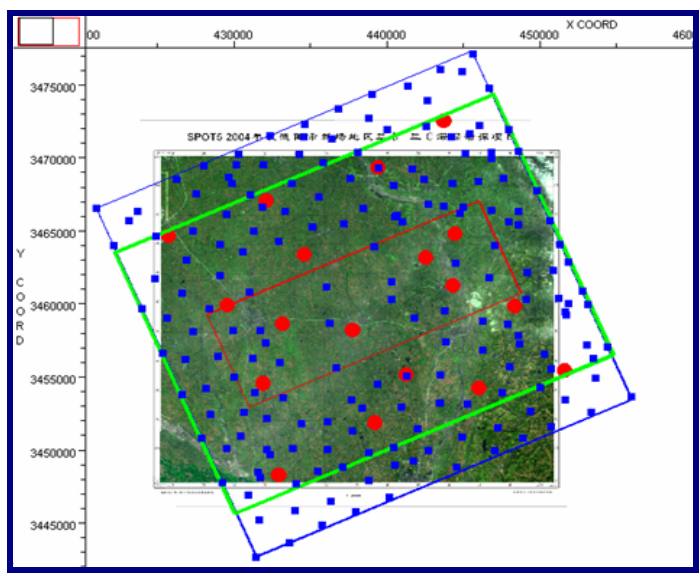

(c) Near surface measurements

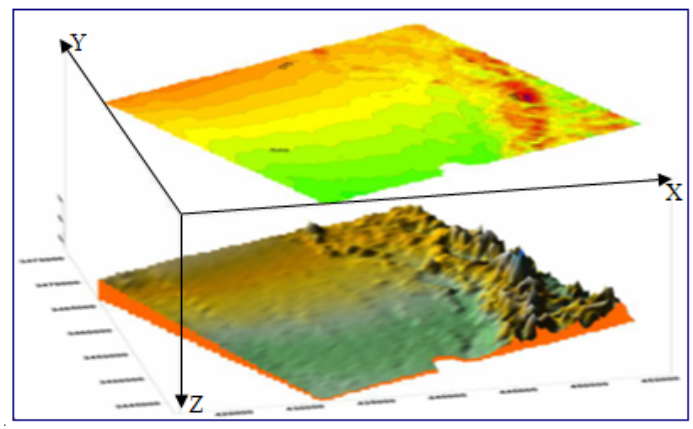

(b) Surface topography

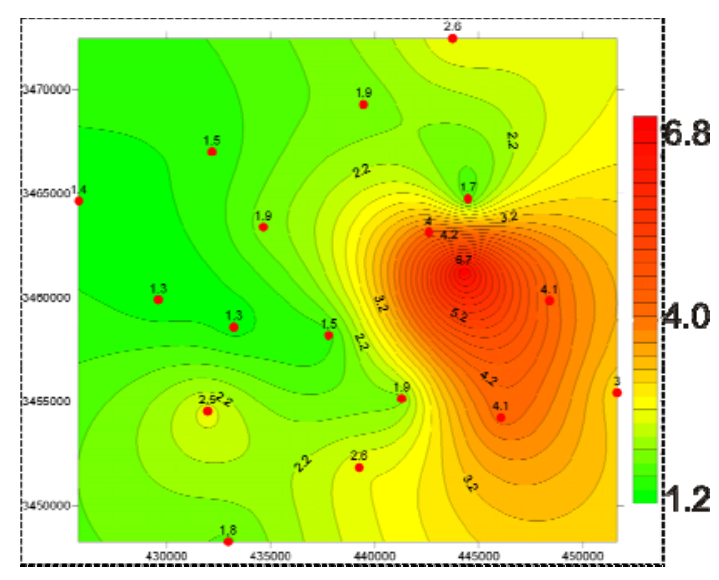

(d) Near surface Vp/Vs model

Figure 4: The acquisition programme: (a) Acquisition layout of the test lines, (b) surface topography, (c) locations of micro-refraction survey marked by the blue points and multicomponent uphole marked by the red points, and (d) the near surface $\mathrm{Vp} / \mathrm{Vs}$ model derived from (c).

The use of digital MEMS sensors has lead to the acquisition of very high quality shear-wave data (Figure 5), and major events can be clearly identified in the original shot records, although some ground roll can be observed. The dominant frequency is about $40 \mathrm{hz}$ for the P-wave and about $20 \mathrm{hz}$ for the converted-wave. There is a significant amount of energy present in the crossline (Y) component, indicating the presence of shear-wave splitting. 

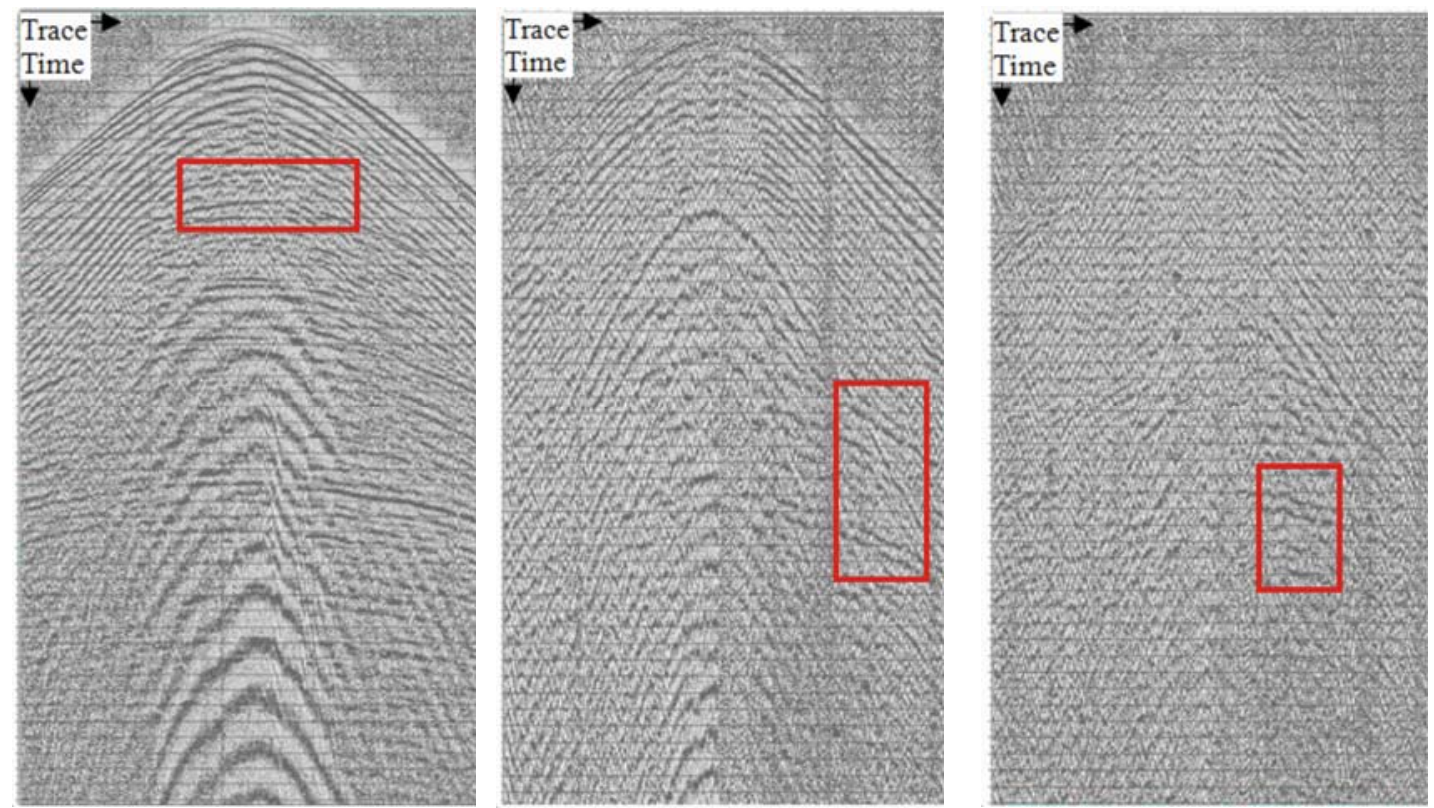

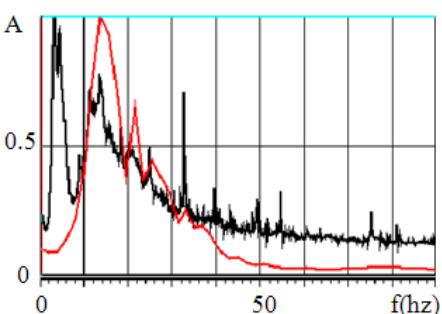

(a) Vertical - Z

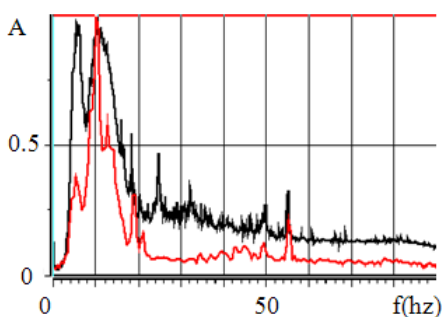

(b) inline horizontal $-\mathrm{X}$

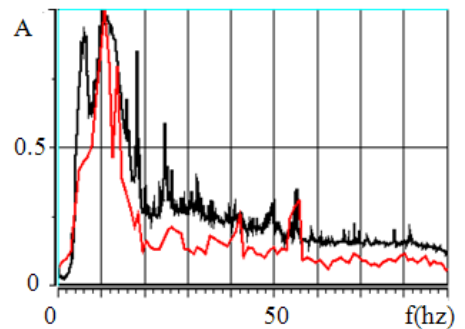

(c) cross-line horizontal-Y

Figure 5. Sample shot records from Line 3 and their corresponding frequency spectra: (a) vertical Z-component, (b) horizontal X-component and (c) horizontal Y-component. The black curves in the spectra are calculated from whole trace, and the red curves are calculated from the selected windows indicated by the red rectangles in the seismograms.

Table 2: Acquisition parameters of the 2D3C experiment

\begin{tabular}{|c|c|c|c|}
\hline Trace interval & $40 \mathrm{~m}$ & Source type & Dynamite \\
\hline Min. offset & $40 \mathrm{~m}$ & Source size & $10 \mathrm{Kg}$ \\
\hline Max. offset & $8640 \mathrm{~m}$ & Explosion Depth & $12 \mathrm{~m}$ \\
\hline Shotpoint interval & $120 \mathrm{~m}$ & Receiver type & $3 \mathrm{C}$ \\
\hline Fold & 36 & Recording system & $408 \mathrm{XL}$ \\
\hline Number of traces & 216 & Sample interval & $2 \mathrm{~ms}$ \\
\hline Interval in array & $4 \mathrm{~m}$ & Record length & $8 \mathrm{sec}$. \\
\hline
\end{tabular}




\section{Data processing}

The main challenge is to obtain quality shear-wave sections with maximum preservation of shear-wave splitting. Generally speaking, there are three main difficulties for processing converted-waves, i.e. noise, statics and non-hyperbolic moveout due to the asymmetric raypath and anisotropy. For this data, the target formation is very deep at over $5000 \mathrm{~m}$, and the offsetto-depth ratio is about one. Therefore, for the target formation, the non-hyperbolic effects are relatively weak. According to Li and Yuan (2003), both the non-hyperbolic moveout and the positioning error due to the asymmetry raypath are small and may be neglected for offsetdepth ratio less than one. Therefore in this case, noise and statics are the main issue for processing the converted-wave data.

The main problem in noise reduction for this data is how to remove the ground roll that is widely present in the data. In conventional data acquisition, array forming is usually used to reduce the ground roll. Here, data are acquired using single-point sensors, and severe ground roll can be observed in the data. We test different techniques for reducing the ground roll, including a deterministic model-based technique (Qian and Zhao, 2003), and the conventional F-K filtering technique. Figure 6 shows the noise-reduction results after applying a modelbased deterministic technique. The model-based technique is very effective in removing the ground roll and other noise, particularly for the near-offset traces.

Our strategy to solve the static problem is through data acquisition. The first step is to build the near-surface P-wave velocity model using refraction surveys, and the second step is to obtain a near-surface $\mathrm{Vp} / \mathrm{Vs}$ model from multicomponent uphole measurements. The nearsurface S-wave velocity model can then be calculated from these measurements. For this, a micro-refraction survey with coverage of $2 \mathrm{kmx} 2 \mathrm{~km}$, totally 200 points was conducted to investigate the P-wave near-surface velocity model, as marked by the blue points in Figure 4c. In addition eighteen multicomponent upholes were recorded for determining the near-surface $\mathrm{Vp} / \mathrm{Vs}$ model, as marked by the red points in Figure 4c. This gave us a very good control of the near surface velocity models. Figure $4 \mathrm{~d}$ shows the derived near-surface $\mathrm{Vp} / \mathrm{Vs}$ model from these measurements for the survey area. The value varies from 1.5 to 6.7, indicating that the nearsurface S-wave velocity model is complicated. Although this increased the cost of the experiment, it did provide a good solution to the notorious shear-wave static problem.

Due to the improved data quality and near-surface data availability, the following seven steps are applied to both the vertical (PP-) and horizontal (PS-) components: 1) geometry loading; 2) static correction; 3) noise attenuation (ground roll); 4) CMP bin for PP, but ACP (Asymptotic conversion point) bin for PS with binning velocity ratio $\gamma=2.0$; 5) velocity analysis, 6) moveout correction; 7) stack. 


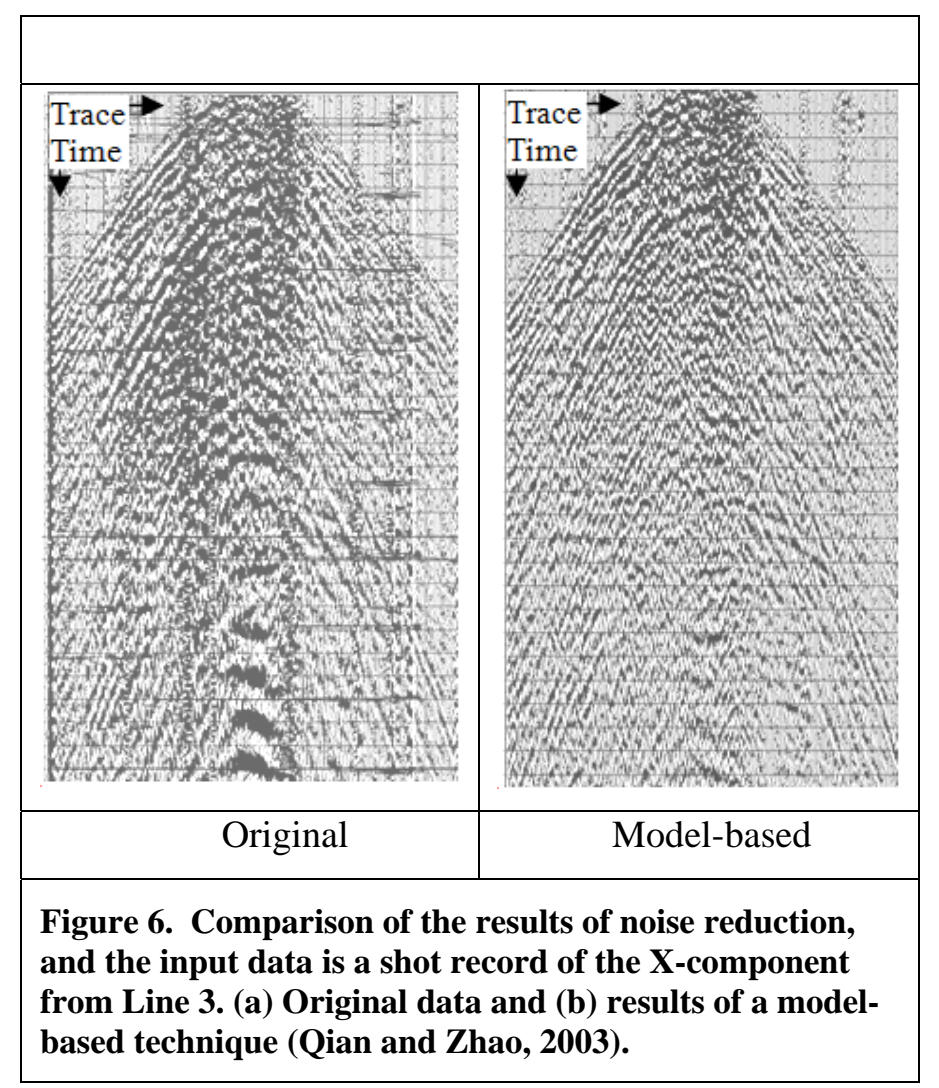

The above processing flow is applied to the three lines separately. The final stacked sections of the vertical Z- and horizontal X-and Y-components for the three lines (L1, L2 and L3) are shown in Figure 7, and all sections are shown in vertical P-wave time for easy comparison. In the zone of interest, as highlighted by the red rectangles, and at the intersection point, the P-wave shows little difference in amplitude and waveform, indicating no-azimuthal variation; there is also no-observable mistie at the well location. However, there are clear misties in the horizontal components at the well locations among the three lines. L3 is of the highest quality; the events are strong and continuous. At the target formation, the events on the $\mathrm{X}$-component are faster than those on the Y-component, and the time delay is about $6 \mathrm{~ms}$ (Figure 7c). L1 is of the poorest quality, but a larger time delay of $20 \mathrm{~ms}$ is observable between the $\mathrm{X}$ and $\mathrm{Y}$ components with the $\mathrm{X}$-component edging forward. L2's quality is slightly better than L1, and there is also a similar amount of time delay between the two horizontal components, but with the Y-component edging forward. This indicates that the fracture strike is close to the direction of L1. This, together with the presence of strong coherent energy in the Y-component, forms the basis of using shear-wave splitting to characterize the fractured reservoir. 

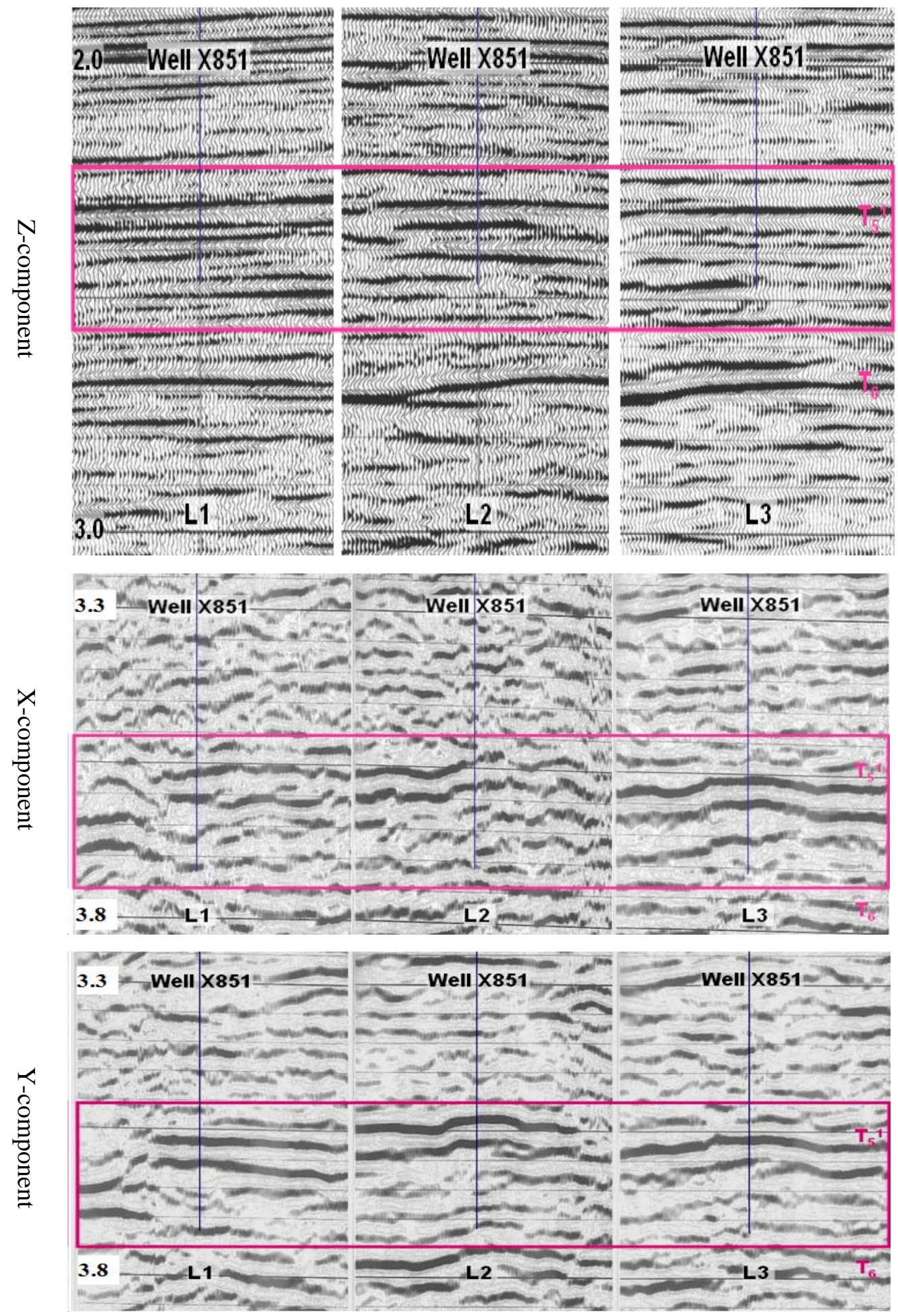

(a) Line L1

(b) Line L2

(c) Line L3

Figure 7. Final stacked sections for Lines (a) L1, (b) L2 and (c) L3. The top row is the vertical Zcomponent, the middle and bottom rows are the horizontal $R$ - and $T$-components, respectively. The red boxes highlight the zones of interest. All sections are displayed in P-wave two-way vertical time. 


\section{Analysis of shear-wave splitting}

In general, except for specific symmetry directions, when a P-S conversion occurs at a reflector and the medium above the reflector is azimuthally anisotropic, the converted shear wave splits into a fast and a slow shear wave, and this is referred to as converted shear-wave splitting. The splitting can be caused by the alignment of microcracks and fractures as well as other small heterogeneities in the reservoirs; the polarization of the fast wave indicates the fracture orientation and the time-delay between the fast and slow waves indicates the fracture intensity. There is a wide interest from the industry to use converted shear-wave splitting to evaluate fractured reservoirs (Li, 1997, 1998; Gaiser and Van Dok 2001; Angerer et al. 2002; Vetri et al. 2003; amongst others). In recent years, it has also become popular to use wideazimuth P-wave seismic data for fracture detection (Wang et al. 2007). Compared with Pwaves, converted-waves can provide more accurate fracture information, in particular, the distribution of fracture density. Here, we provide a quantitative analysis of shear-wave splitting using Line L3, focusing on the extraction of the time-delay between the split shearwaves.

Analysis of shear-wave splitting often involves rotation of the recording coordinate system. Most of the rotation algorithms for shear-wave splitting, such as Alford rotation (1986) and the linear transform technique ( $\mathrm{Li}$ and Crampin 1993), require multi-azimuth distribution. However, the converted-wave data used in this study are acquired with a 2D configuration and single-azimuth source and receiver distribution. For single-azimuth data, a rotation scanning procedure is often needed that searches for optimum solutions according to certain criteria. One of the common criteria is the similarity of waveforms between the fast and slower shearwaves. Here we adopt a criterion that maximizes the separation of the fast and slow shearwaves, following the approach of Yuan (2001).

Once the polarization of the fast wave is determined, split shear-waves can be separated, and time delay between the fast and slow shear waves can be estimated using cross-correlation. For quantitative analysis of the time-delay, we construct a time-delay spectrum that allows the picking of time delays as a function of the vertical travel time, yielding a time-delay section for interpretation purposes. The time-delay spectrum can be constructed by scanning over vertical time using the correlation method. For each trace pair of the fast and slow split shear-waves, we use a sliding time-window to compute the correlation coefficients within the window, which produces a time-delay spectrum. In order to maintain the characteristics of shear-wave 
splitting, the two horizontal components are processed using the same processing flow as described previously, and the analysis is performed on the stacked data as shown in Figure 7c.

Figure 8a shows the time-delay spectra from selected CDP locations of Line 3, from which a time-delay section can be obtained, as shown in Figures 8b and 8c. Figure 8b shows the accumulative time delays directly picked from the time-delay spectra, and the colour indicates time delays in milliseconds. For interpretation, the interval time delay is often used, and this can be obtained by calculating the time-delay gradient along the vertical axis in Figure 8b, and the results are shown in Figure 8c, where the colour bar indicates percentage shear-wave splitting. Figure 9 shows the interpretation results of the time-delay gradient section, superimposed on the stacked seismic section. As shown in Figure 9, areas of high gas accumulation show a significant amount of splitting (well X851). The splitting anomalies seem to be good indicators of gas accumulation, and can be used to delineate the fractured reservoirs.

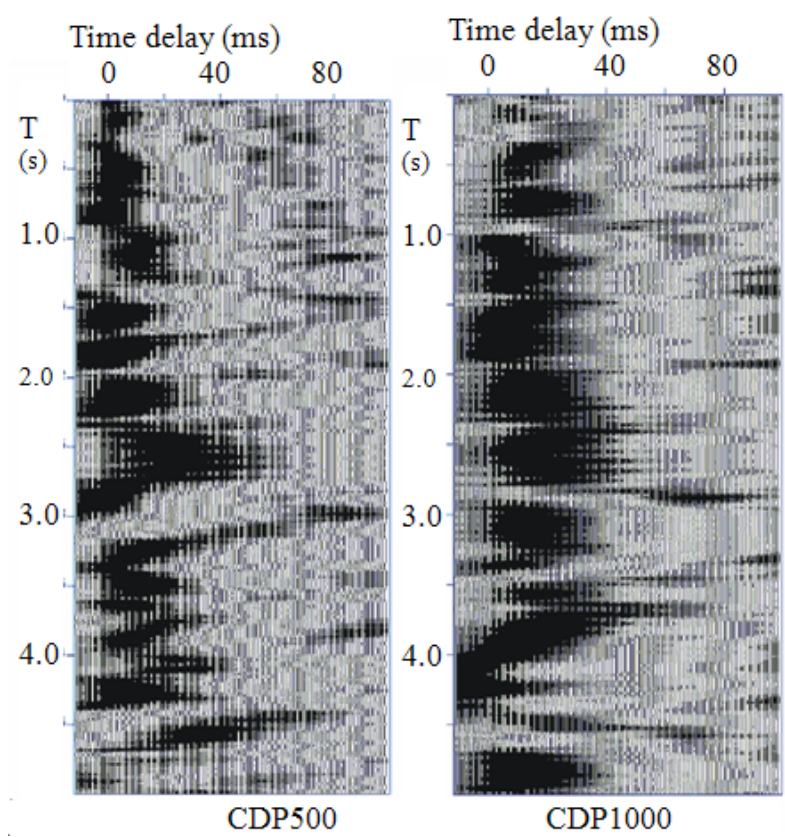

(a) Time-delay spectra

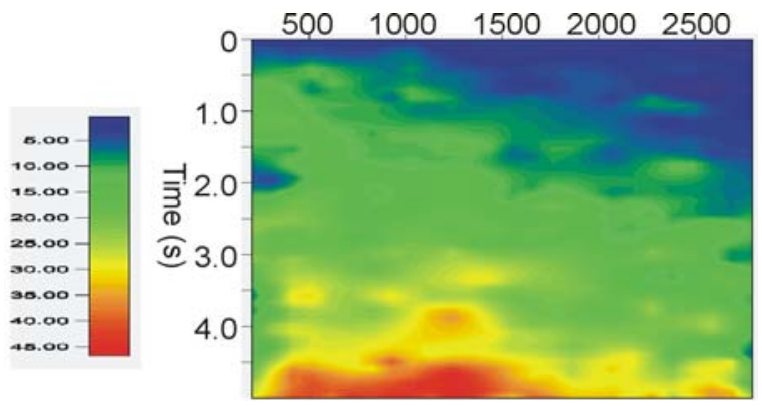

(b) Accumulative delay

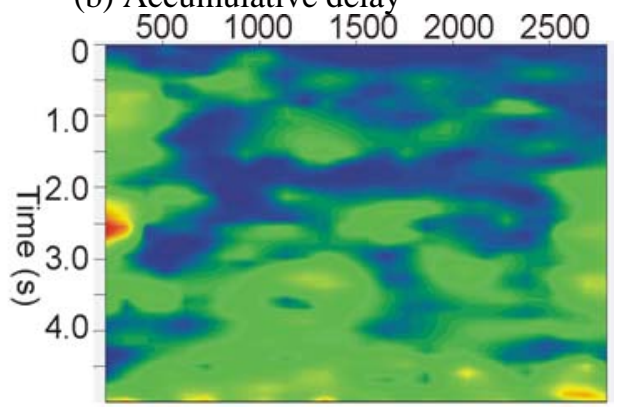

(c) Time-delay gradient

Figure 8. Calculated time-delay spectra of the split shear-waves for Line L3; (b) accumulative time delays picked from the spectra in (a), and the colour bar indicates time delay in milliseconds; (c) time-delay gradient calculated from (b), and the colour bar indicates percentage shear-wave splitting. 


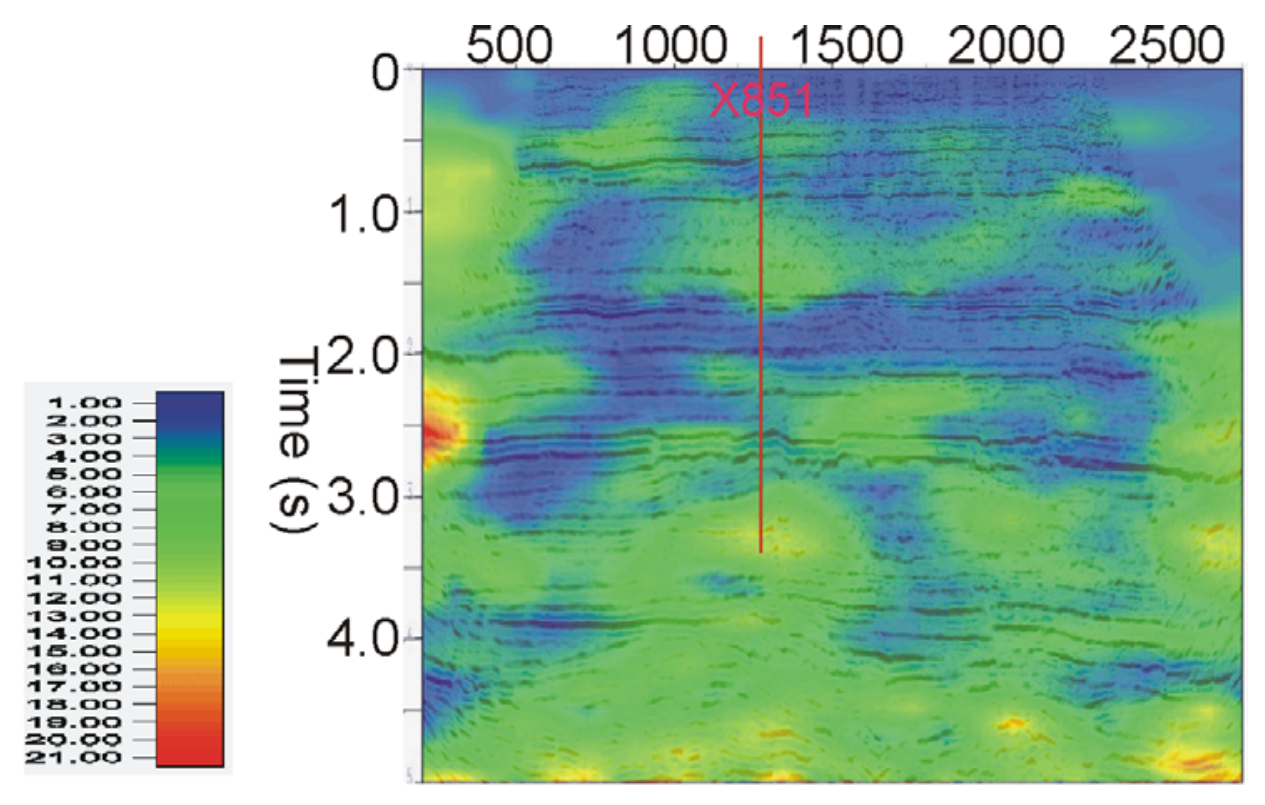

Figure 9. Interpretation results on the time-delay gradient section superimposed by the corresponding seismic section. X851 is the most productive gas well in the study area, which is drilled into a zone with more than $15 \%$ shear-wave anisotropy. The colour bar on the left indicates percentage shear-wave splitting.

\section{Analysis of P- and S-wave impedance variations}

Impedance inversion is widely used for fluid and lithology prediction. There are three common attributes: acoustic impedance, shear-wave impedance and elastic impedance. Various other attributes can be derived from these three attributes, such as Poison's ratio, Lame constants, etc. In theory all these attributes can be inverted from prestack P-wave data with calibration from borehole logs. However, shear-wave impedance is generally less accurate when inverted from prestack P-wave data. One of the benefits of acquiring multicomponent data is that shear-wave impedance can be more accurately inverted from the horizontal components. Here, we will evaluate the various impedance attributes for delineating the fractured gas sands.

First, we analyze the variation in the $\mathrm{Vp} / \mathrm{Vs}$ ratio as calculated from the sonic logs, which provides the basis for impedance inversion. The presence of gas reduces P-wave velocity, whilst its effects on the S-waves are relatively small (Jiang et al., 2004), since the S-wave is 
generally less sensitive to the presence of fluids than the P-wave. This usually leads to clear anomalies in the $\mathrm{P}$ - and $\mathrm{S}$-wave velocity ratio ( $\mathrm{Vp} / \mathrm{Vs}$ ratio) for gas sand. Note that theoretically, gas-filled cracks slightly increase percent of shear-wave anisotropy in offvertical directions (Crampin, 1993).

Figure 10 shows the sonic logs, the calculated $\mathrm{Vp} / \mathrm{Vs}$ ratio and its correlation with lithology. We can see that there are clear anomalies associated with the gas sand, as indicated by the yellow colours in Figure 10. These observations provide the basis for the use of multicomponent data to discriminate the gas-bearing reservoirs, and in particular for the following seismic inversion studies.

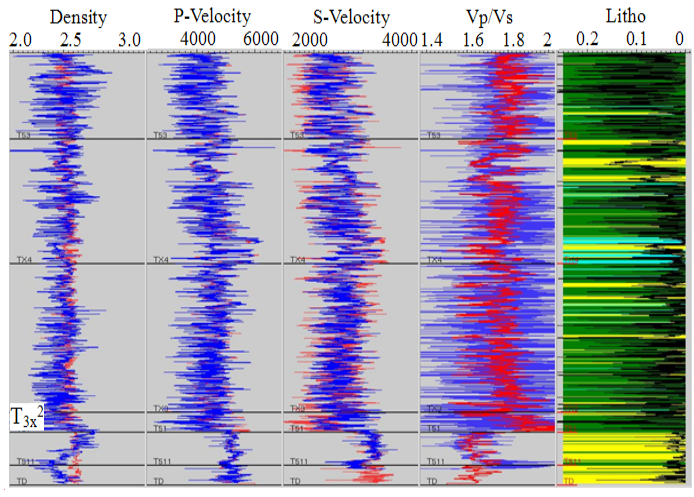

(a) Sonic logs

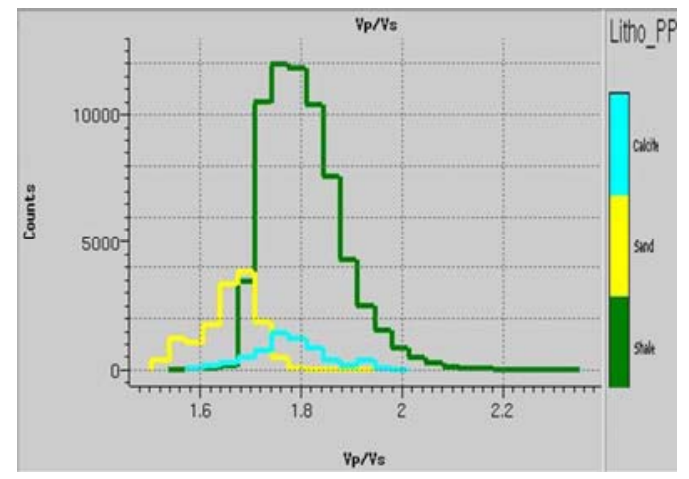

(b) $\mathrm{Vp} / \mathrm{Vs}$ and lithology

Figure 10: (a) Sonic logs from borehole X851, where $T_{3 x} 2$ marks the target formation corresponding to Figure 1, and (b) correlation of $\mathrm{Vp} / \mathrm{Vs}$ ratio with lithology. Yellow stands for gas sand, green for shale and light blue for calcite.

Figures 11, 12, 13 and 14 show the inversion results at the zone of interest for all three lines L1, L2 and L3. These include acoustic impedance sections inverted from the Zcomponents (Figure 11), shear impedance sections inverted from the horizontal X-components (Figure 12), elastic impedance at an incidence angle of $30^{\circ}$ (Figure 13) and the derived Vp/Vs ratio sections from the inverted acoustic and shear impedance (Figure 14). 


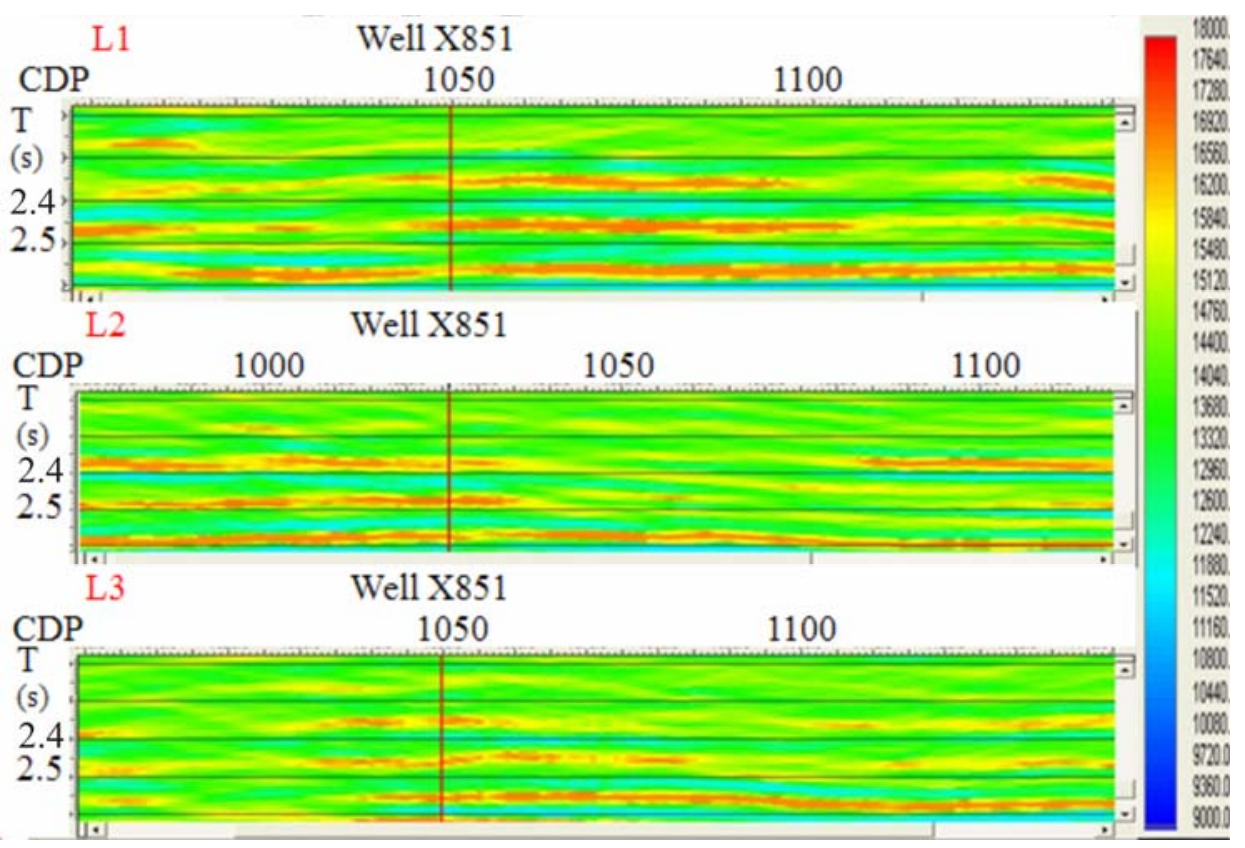

Figure 11. Zoomed acoustic impedance sections at the zone of interest inverted from the Z-components for lines L1 (top), L2 (middle) and L3 (bottom)..

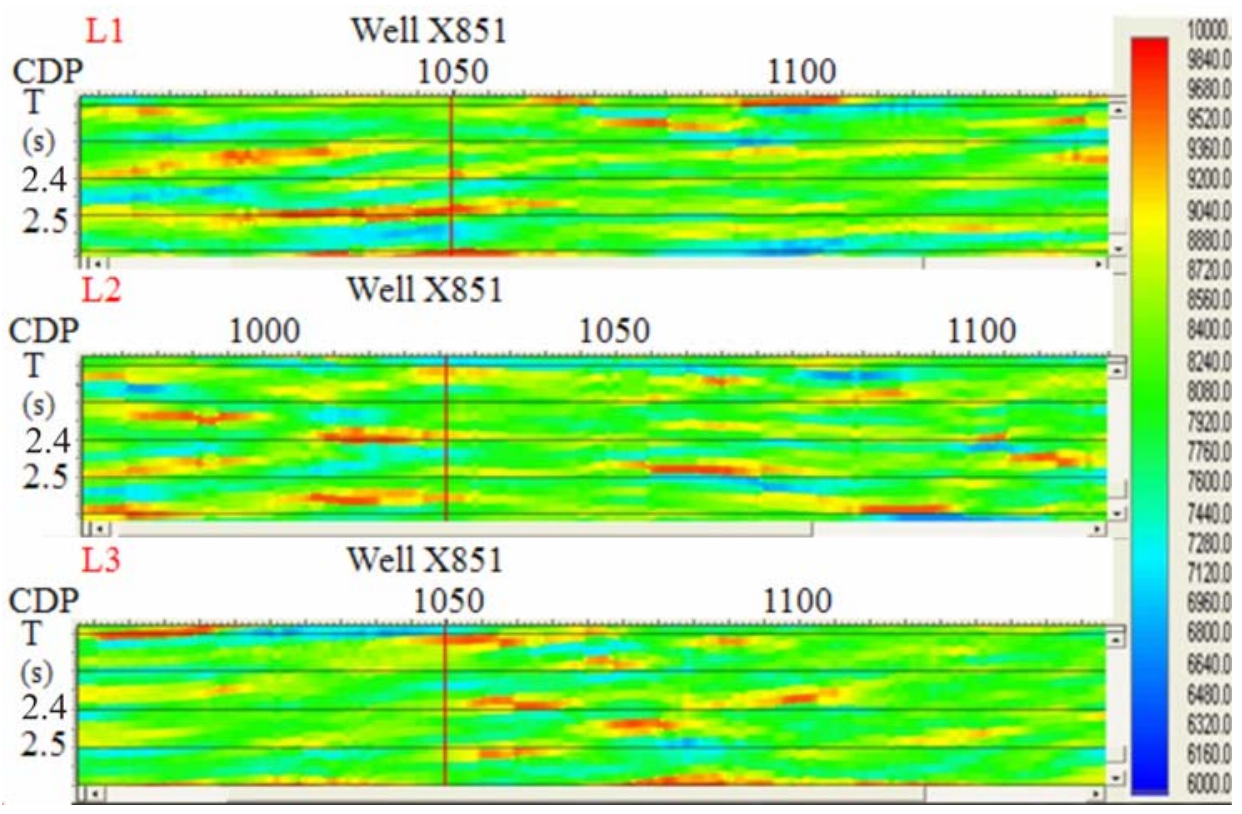

Figure 12. Zoomed shear-wave impedance sections inverted from the inline $X$ components for lines L1 (top), L2 (middle) and L3 (bottom). 


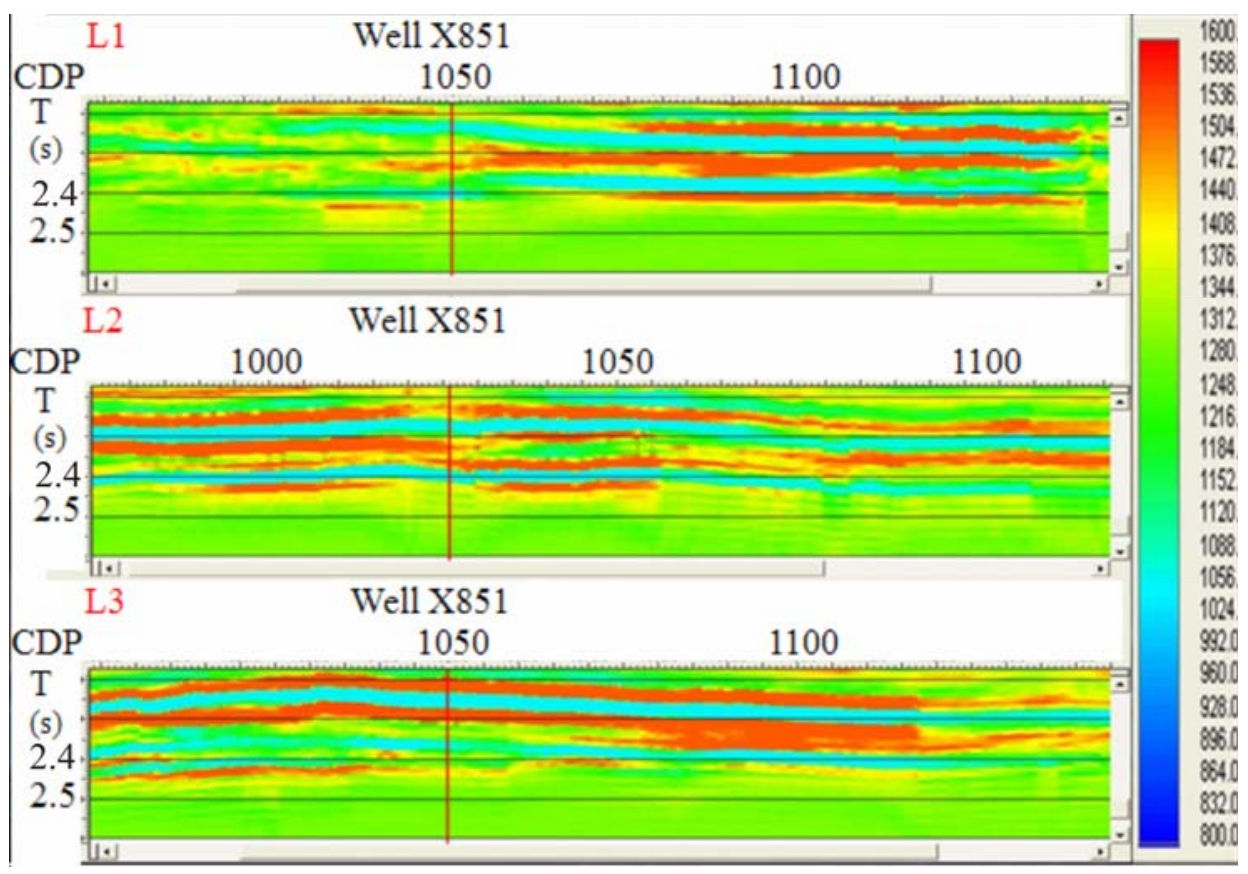

Figure 13. Zoomed elastic impedance sections inverted from the Z-components for lines L1 (top), L2 (middle) and L3 (bottom) at incidence angle $30^{\circ}$.

There are two main pay beds in the study area, pay bed $\mathrm{TX}_{2} 4$ as encountered by well X851 at 2.4 seconds, and $\mathrm{TX}_{2} 6$ as encountered by well X853 at 2.5 seconds. We can see a clear low impedance (blue colour) in the acoustic impedance sections (Figure 11) and elastic impedance sections (Figure 13), whist a relative high impedance (an orange-yellow colour) in the shearwave section (Figure 12). This leads to low blue colour in the $\mathrm{Vp} / \mathrm{Vs}$ ratio sections (Figure 14) which agrees with the sonic logs in Figure 10. Other subtle variations can also been observed among the three lines, and these variations can be attributed to various reasons such as anisotropy, noise, near-surface conditions. However, the consistent variation in the warm and cold colour at the impedance sections confirms the benefit of using multicomponent data for characterizing these reservoirs. A combined attribute based on absorption and velocity dispersion of the P- and S-waves (Chapman et al. 2003) may also be derived from the data, as shown in Figure 15, where a clearer correlation between the anomalies and the pay beds can be observed. 


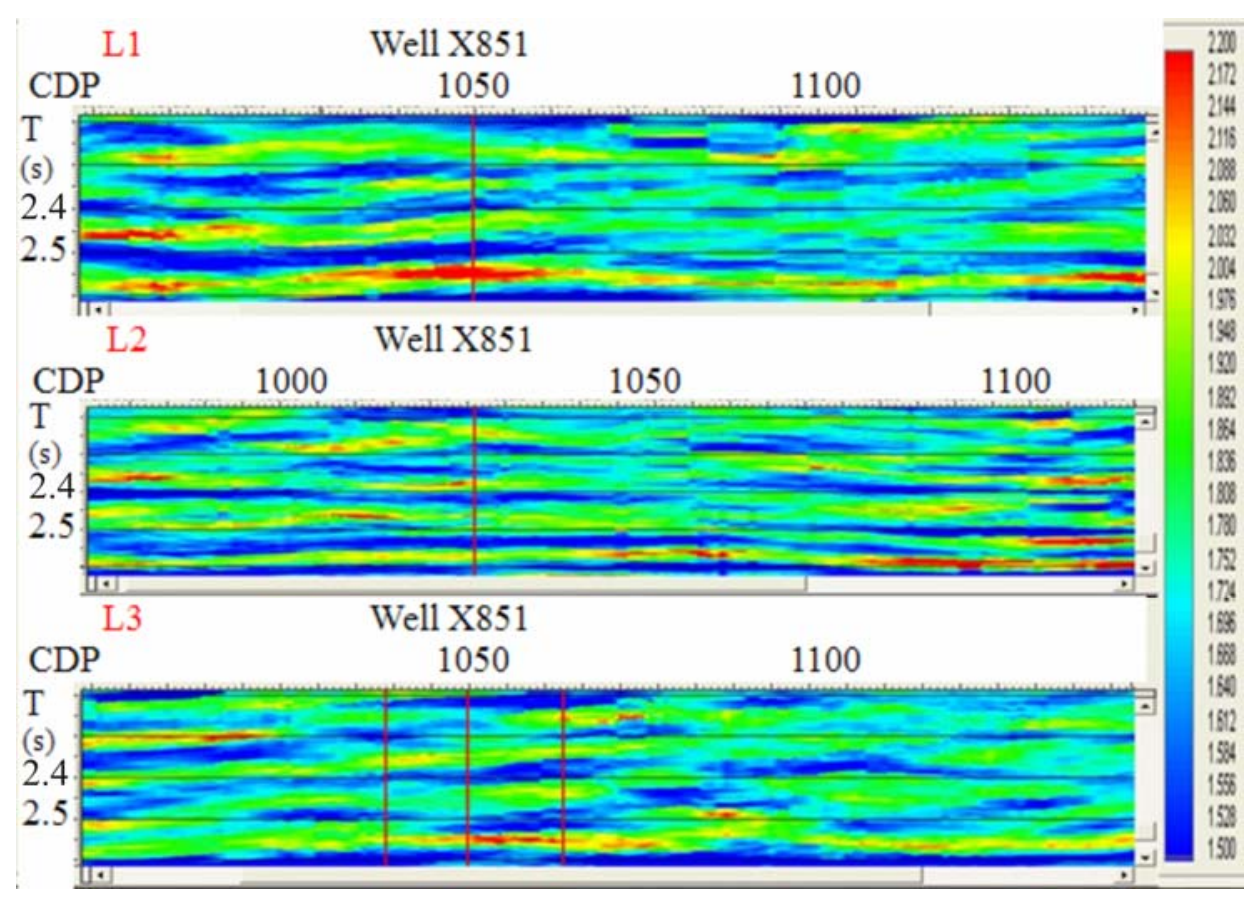

Figure 14. Calculated Vp/Vs ratio sections from Figures 11 and 12 at the zones of interest for lines L1 (top), L2 (middle) and L3 (bottom)..

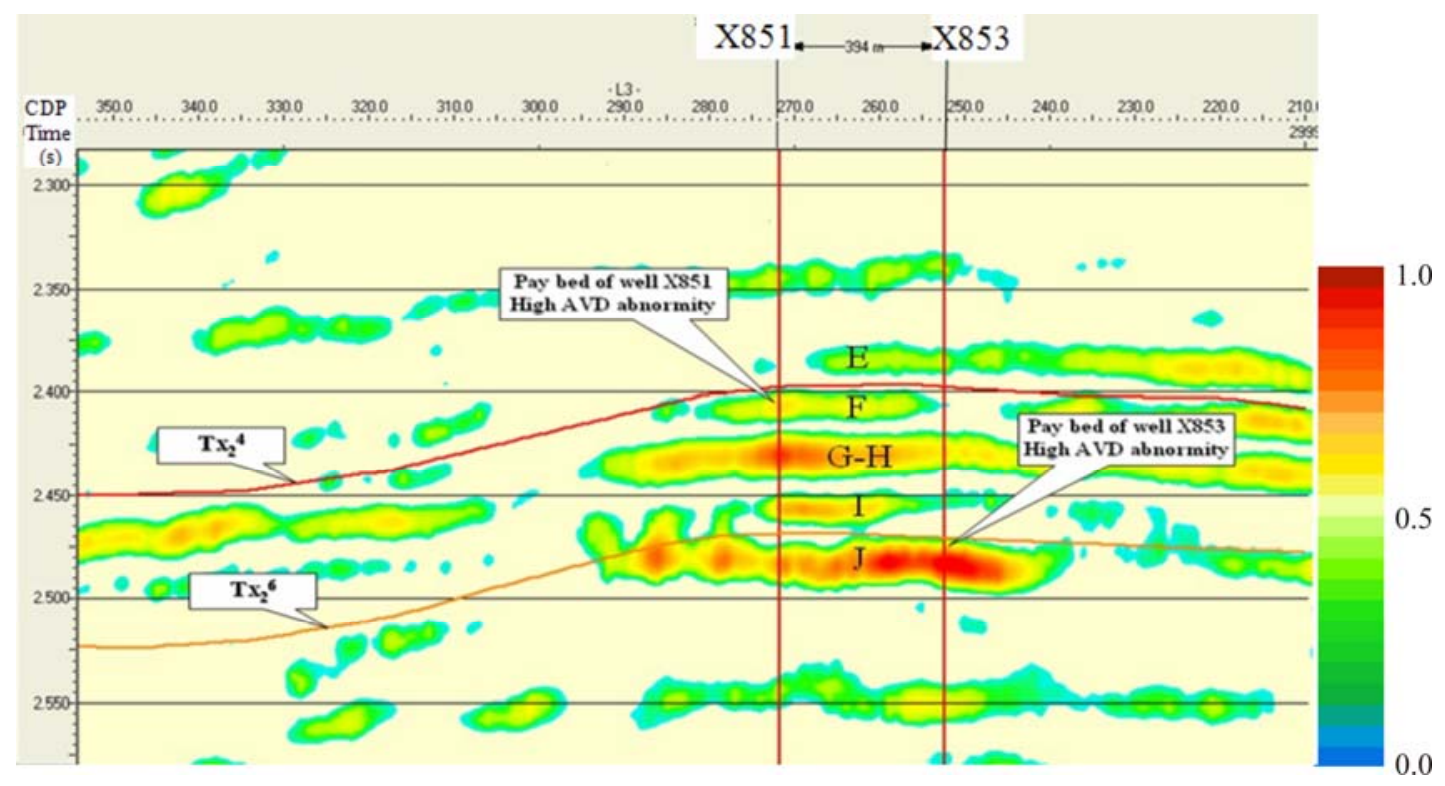

Figure 15: Absorption and velocity dispersion (AVD) calculated from the P- and S-wave data for Line L3. 


\section{Discussion and conclusions}

We have shown a case study where multicomponent data are used for delineating fractured gas reservoirs in tight sandstone formations. The multicomponent data are acquired using digital MEMS (micro-electro-mechanic-system) sensors. Although good converted-wave data are obtained, the single-sensor data are contaminated with strong ground roll. The ground roll can be effectively removed by a model-based noise reduction technique (Qian and Zhao, 2003). The target formation is buried at a depth of more than $5000 \mathrm{~m}$, and consequently the nonhyperbolic moveout effects are not very significant and simple straightforward processing can be applied to the converted-wave data. Detailed refraction surveys and wide recording of multicomponent uphole time are very beneficial for providing an accurate solution to the shear-wave static problem, although they will increase the acquisition cost.

In terms of gas reservoir response, the PP- and PS-attributes show consistent anomalies that diagnose the presence of fractured gas sands. First, significant shear-wave splitting is observed at the target formation, and regions of high gas accumulation with high productivity can be correlated with the amount of shear-wave splitting. Secondly, low acoustic and elastic impedance inverted from the P-wave data can be observed at the zone of interest, and a consistent low $\mathrm{Vp} / \mathrm{Vs}$ ratio can also be observed at the fractured gas sands. This is demonstrated in both the $\mathrm{Vp} / \mathrm{Vs}$ sections as calculated from the $\mathrm{P}$ - and $\mathrm{S}$-wave impedance attributes and the $\mathrm{Vp} / \mathrm{Vs}$ ratio from borehole sonic logs. Thirdly, a combined attribute measuring the absorption and velocity dispersion also shows consistent anomalies associated with the gas sands. As a result, the gas reservoirs can then be delineated from joint PP- and PSwave analysis. The results agree with the drilling results in the study area. This provides another convincing example demonstrating the benefit of multicomponent seismic data.

\section{Acknowledgements}

We thank SinoPec Southwest Ltd. for permission to show the data. We thank Mr. Zhang Yonggang for coordinating this project. This work is supported by the SinoPec international collaboration programme and SinoPec Southwest Ltd. through the Edinburgh Anisotropy Project (EAP) of the British Geological Survey (BGS), and is published with the approval of all project partners and the Executive Director of BGS (NERC).

\section{References}

Alford, R., 1986. Shear data in the presence of azimuthal anisotropy: Dilley, Texas: 56th Annual International Meeting, SEG, Expanded Abstracts , 476-479. 
Angerer, E., Crampin, S., Li, X.Y. and Davis, T.L., 2002. Processing, modelling and predicting time-lapse effects of overpressured fluid-injection in a fractured reservoir Geophysical Journal International, 149, 267-280.

Calvert, A.S., Novak, J.M., Maher, J., Burch, D.N., Bird, D., Larson, R., 2005. A tale of two surveys: experiences processing two similar but different land 3D-3C MEMS surveys. $75^{\text {th }}$ SEG meeting, Houston, USA, Expanded Abstracts, 975-978.

Chapman M., Maultzsch S., Liu E. and Li X.-Y. 2003. The effect of fluid saturation in an anisotropic multi-scale equant porosity model. Journal of Applied Geophysics, 54, 191202.

Crampin, S., 1993. A review of the effects of crack geometry on wave propagation through aligned cracks. Canadian Journal of Exploration Geophysics, 29, 3-17.

Gaiser, J. and Van Dok, R., 2001, Green River basin 3D/3C case study for fracture characterization: Analysis of PS-wave birefringence, 71st Ann. Internat. Mtg: Soc. of Expl. Geophys., 764-767.

Jiang, C., Chen, S., Zhang, E. and Zhong, D. 2004. Methodology and application of seismic prediction of gas-bearing volcanic reservoir: 74th SEG Meeting, Denver, Expanded Abstracts, 1595-1598.

Li, X.-Y., 1997. Fractural reservoir delineating using multicomponent seismic data. Geophysical Prospecting, 54, 39-64

Li, X.-Y., 1998. Processing PP and PS waves in multicomponent sea-floor data for azimuthal anisotropy: theory and overview, Proceedings of the Eighth International Workshop on Seismic Anisotropy (Revue De L'Institut Francia du Petrole), 53, 607-620

Li, X.-Y. and Crampin, S., 1993. Linear transform techniques for analysing split shear-waves in four-component seismic data. Geophysics, 58, 240-256

Li, X.-Y., Dai, H. and Mancini, F., 2007. Converted-wave imaging in anisotropic media: theory and case studies. Geophysical Prospecting, 55, in press.

Li, X.-Y. and Yuan, J. 2003. Converted-wave moveout and conversion-point equations in layered VTI media: theory and application. Journal of Applied Geophysics, 54, 297318.

Qian, Z. and Zhao, B., 2003, A regular noise elimination method for prestack 3D seismic data: 73rd SEG Meeting, Dallas, Expanded Abstracts, 2040-2043.

Stewart R.R., Gaiser J.E., Brown R.,J. and Lawton D.C. 2002. Converted-wave seismic exploration: Methods. Geophysics 67, 1345-1363.

Vetri, L., Loinger, E., Gaiser, J., Grandi, A. and Lynn, H., 2003, 3D/4C Emilio: Azimuth processing and anisotropy analysis in a fractured carbonate reservoir. The Leading 
Edge, 22, 675-679.

Wang, S., Li, X.-Y., Qian, Z., Di, B. and Wei, J. 2007. Physical modelling studies of 3D Pwave seismic for fracture detection. Geophy. J. Int. 168, 745-756.

Yuan, J. 2001, Analysis of four-component sea-floor seismic data for seismic anisotropy. PhD Thesis, University of Edinburgh. 